\title{
Reliability and validity of new evaluation methods using static surface electromyography in persons with neck pain
}

\author{
Go-Eun Kim ${ }^{\mathrm{a}}$, Dong-Uk Yun ${ }^{\mathrm{b}}$, Yu-Ju An ${ }^{\mathrm{b}}$, Dae-Sung Park ${ }^{\mathrm{b},{ }^{\prime}}{ }^{\dagger}$, Joo-Hyun Ham ${ }^{\mathrm{a}, *}, \dagger$ \\ ${ }^{a}$ Department of Chiropractic, The Graduate School of Health Promotion, Hanseo University, Seosan, Republic of Korea \\ ${ }^{\mathrm{b}}$ Department of Physical Therapy, College of Medical Science, Konyang University, Daejeon, Republic of Korea
}

\begin{abstract}
Objective: The aim of this study was to evaluate the validity and reliability of using static surface electromyography (sEMG) on persons with neck pain and in healthy adults.

Design: Cross-sectional study.

Methods: Twenty-two female participants with neck pain and thirty healthy adults in the age group of 20-65 years were recruited in this study. To evaluate the validity and reliability of sEMG in subjects with neck pain, the subjects'characteristics were recorded and the Visual Analog Scale (VAS) and Neck Disability Index (NDI) were examined in addition to sEMG and algometer tests being carried out on the subjects. The site for using the sEMG and algometer was the upper trapezius. sEMG test-retest reliability was measured by intraclass correlation coefficients (ICCs). Independent t-tests were used to analyze the differences in the dependent variables between subjects with neck pain and healthy adults. The Pearson correlation coefficient was used to examine the linear relationship between measured variables.
\end{abstract}

Results: sEMG and algometer tests were reliable according to the test-retest reliability results in subjects with neck pain and healthy adults ( $\mathrm{ICC}=0.815-0.979)$. The results of this study showed that there were significant differences in respect to age, VAS, sEMG and algometer tests between persons with neck pain and healthy adults $(p<0.05)$. The VAS and NDI were statistically correlated with sEMG and algometer results $(p<0.05)$.

Conclusions: In this study, we investigated the clinical usefulness of the static sEMG test in evaluating the pain scale of persons with neck pain with high reliability and validity.

Key Words: Diagnosis differential, Diagnostic techniques and procedures, Electrodiagnosis, Neck injuries

\section{Introduction}

Seventy-one percent of the world's population has a high incidence of experiencing neck pain at least once in their lifetime. It has confirmed that $45.5 \%$ of a total of 512 workers in the office have compliants of neck pain, and in 50\%$85 \%$ of those with neck pain, $47 \%$ have chronic symptoms in which the symptoms are not completely resolved [1]. Pati- ents with pain often complain of subjective symptoms, such as muscle stiffness and tension along with pain, and the main clinical symptoms lead to an imbalance of the soft tissues around the neck and shoulders, and painful areas on the neck, shoulder, back, scapula, fatigue headaches, and restriciton of range of motion, but in severe cases, it is associated with functional disability of the neck, such as muscle weakness, muscle atrophy, and muscle tension, depending

Received: 3 October, 2018 Revised: 31 October, 2018 Accepted: 1 November, 2018

Corresponding author: Dae-Sung Park (ORCID https://orcid.org/0000-0003-4258-0878)

Department of Physical Therapy, College of Medical Science, Konyang University, 158 Gwanjeodong-ro, Seo-gu, Daejeon 35365, Republic of Korea Tel: 82-42-600-8456 Fax: 82-42-600-8408 E-mail: daeric@konyang.ac.kr

Corresponding author: Joo-Hyun Ham (ORCID https://orcid.org/0000-0002-3030-5820)

Department of Chiropractic, The Graduate School of Health Promotion, Hanseo University, 46 Hanseo 1-ro, Haemi-myeon, Seosan 31962, Republic of Korea Tel: 82-41-660-1098 Fax: 82-41-660-1098 E-mail: dcham72@hanseo.ac.kr

*These authors contributed equally to this work as corresponding authors.

${ }^{+}$These two authors contributed equally to this work as co-first author.

(c) This is an Open-Access article distributed under the terms of the Creative Commons Attribution Non-Commercial License (http://creativecommons.org/licenses/ by-nc/4.0) which permits unrestricted non-commercial use, distribution, and reproduction in any medium, provided the original work is properly cited.

Copyright (c) 2019 Korean Academy of Physical Therapy Rehabilitation Science 
on the site of the nerve root lesion [2]. In clinical practice, to measure the degree of disability and quality of life in relation to the pain of the neck, the Neck Disability Index (NDI) Questionnaire, the Visual Analog Scale (VAS), and the Numerical Rating Scale Subjective measurements are generally used [3]. However, since these instruments cannot quantify pain objectively, electromyography (EMG) is used to measure the muscle activity of a specific muscle and a pressure guage is used to analyze the threshold of pain [3].

Recently, studies have been conducted to analyze pain levels by observing the voltage level and directional pattern of the spinal muscles using the muscular electrical resistance of static surface EMG (sEMG) [4]. Static sEMG is a surface electrode scanner that records electrical resistance in resting and active states by characterizing the electrical potential of a particular muscle [5]. Using the same technology as electrocardiogram and EMG, the muscle tension level of wide muscles is recorded using single or multiple electrodes which are very sensitive to movement. The relatively low frequency band and low signal degradation function represent the muscular muscle tension level as a bar-shaped stress score [6,7]. In particular, static sEMG is a tool for evaluating muscle tension, which is used in persons with back pain symptoms such as spasms, tenderness, limited range of motion, postural disorders, intervertebral disc disease, soft tissue damage, and neuromuscular disorders, and it is used as a diagnostic tool for patients with motor control disorders [5]. In addition, static sEMG diagnoses muscle imbalance, identifies the underlying causes of pain, and confirms the function and condition of the spinal nerve muscles of the patient [7].

In previous studies, there were statistically significant correlations between static contraction and pain in the resting state and static contraction of patients with chronic cervical pain using sEMG, but there was no significant correlation in the resting state [8]. As a result of examining the correlation between muscle activity, pain intensity, and dysfunction of the neck and chest by providing neck movement tasks to patients with chronic cervical pain and normal persons, there was a significant correlation between muscle activity and dysfunction in persons with neck pain [9]. Needle EMG used in previous studies have caused pain during examinations and surface electromyograms were mostly used on subjects with muscle activity values analyzed during an active state. Therefore, static sEMG tests are considered to be more useful for visualizing the muscular signals from a resting state. Static sEMG showed high reliability in the test-retest measurements of spinal fatigue [10] and it exhibited reliability and validity in distinguishing healthy and back pain patients [11]. However, static sEMG has been studied mainly in patients with back pain, and there is a lack of research on the relationship with the evaluation tools used to evaluate pain in clinical practice.

Therefore, the purpose of this study was to determine the reliability of the test method by analyzing the difference between static sEMG and the pressure gauge of each subject in order to test the usefulness of the static sEMG test method for assessing those with cervical pain and healthy adults. In addition, this study was conducted to evaluate the relationship between static sEMG results, the VAS, the NDI, and the pressure gauge system. In this study, if the reliability and validity of static sEMG can be verified, it could be suggested as a useful measurement tool for the assessment of cervical pain.

\section{Methods}

\section{Subjects}

This study was conducted between September 30th and October 27th, 2018 and included a total of 52 patients with 22 female patients between the ages 20-65 year old who had been diagnosed with neck pain from a neurologist in Daejeon Raphael Neurology, and 30 healthy adults.

Those who have sustained cervical pain for more than 3 months, have been diagnosed with a cervical pain from a specialist, and have complained of unilateral pain that is consistent with the patient's pain site were included while those with orthopedic problems, neurological injuries, and serious surgical injuries were excluded.

For healthy adults, those with a score of 15 or less on the NDI questionnaire were selected while those with orthopedic problems, neurological injuries, and serious surgical injuries were excluded. In order to distinguish between nonmalignant and non-malignant tumors, persons with a score of 15 or less on the NDI questionnaire were selected, and those with orthopedic problems, neurological injuries, and serious injuries were excluded. In addition, all subjects provided their informed consent after the explanation of the purpose and procedures of this study were given. This study was approved by the Institutional Bioethics Committee of Konyang University (IRB No. KYU-2018-091-01).

\section{Measurement}

The general characteristics of subjects (age, height, and 
weight), VAS, NDI, static sEMG, and algometer results were examined. The assessment site was located at the site of the upper trapezius muscle to the midpoint between the seventh cervical vertebrae and the acromion process where static sEMG and pressure goniometry was performed on both sides [12]. The examination time for each subject was approximately 20 minutes. The subjects were allowed to rest for 15-20 minutes before the start of the examination process.

\section{Visual Analog Scale}

The VAS is used to determine the subjective pain level of the patient. On a $10-\mathrm{cm}$ line, the left end represents a state of no pain at all ( 0 point), and the right end refers to the maximum pain intensity (10 points) $[13,14]$.

\section{Neck Disability Index}

The NDI is the most commonly used questionnaire to assess cervical spine anomalies and was originally developed to assess the severity of everyday life in persons with severe cervical pain, especially for patients with whiplash trauma [15]. The NDI has 10 items with a total score of 50 points. The lower the score, the lower the degree of dysfunction. The intraclass correlation coefficient (ICC) value of the testretest reliability of this instrument is 0.93 , and the Cronbach alopha value is 0.82 [15].

\section{Static surface electromyography}

Static sEMG was performed with the Myovision EMG 4000 (Precision Biometrics/Myovision, San Carlos, CA, USA). Static sEMG is a wireless device consisting of two joysticks with five electrodes, each of which is designed to record muscle activity levels at the vertebral level where the joystick is located [16]. As a non-invasive device, static sEMG signals are quantified and the actual data is measured in $\mathrm{mV}$ and recorded on the monitor, indicating the amplitude of muscle activity during the measurement time [16]. This equipment uses a frequency filter of $20-500 \mathrm{~Hz}$ and the band-pass filter is used to maximize the continuous range and reduce the possibility of false negatives. The range of scanning is $0.08-200 \mathrm{mV}$ [7]. Prior to the assessment, the electrode containing the disposable aseptic conductive medium was pressed onto the pad on which the joystick was placed, and hair was removed from the assessment site, and the skin was cleansed with an alcohol wipe. The assessment was performed with the subject in sitting position in a chair with the hip and knee joints flexed to $90^{\circ}$ and hands placed onto the knees while looking straight ahead [17]. The measurement site was from the upper trapezius to the midpoint between the seventh cervical vertebrae and the acromion process where joystick electrodes were placed on the top of both right and left sides, which were evaluated simultaneously.

The mean value was obtained from three consecutive measurements from each assessment site (Figure 1).

\section{Algometer}

The Commander Algometer (JTECH Medical, Salt Lake City, $\mathrm{OH}, \mathrm{USA}$ ) was used to evaluate the pressure sensitivity of the pain site. The pressure gauge system is an objective pain assessment method, which is useful for measuring pain points using pressure thresholds, and can quantify the exact location of the pain and the pressure sensitivity of the muscles [18]. The algometer consists of a measuring instrument, a knob, and a recorder. When the strap connected to the recorder is worn on the wrist, the knob is grasped with one

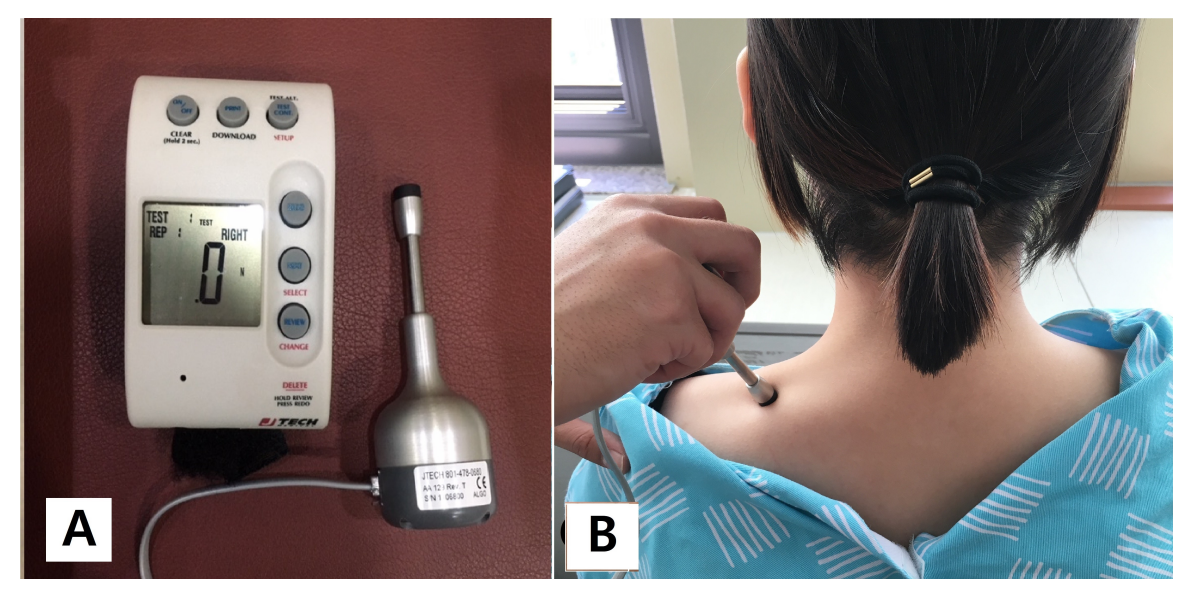

Figure 1. (A) Algometer. (B) Measured upper trapezius. 
hand, and the pressure is applied vertically to the measuring point at a rate of $1 \mathrm{~kg} / \mathrm{s}$ [12]. The measuring instrument was $1 \mathrm{~cm}^{2}$ in diameter and after the time the subject cried ' $\mathrm{Oh}$ ' with pressure application, which indicated the start of the pain sensation, the presssure reading was recorded (Figure 2). The posture and position of the measurement site were the same as those for the static sEMG, and the average of the results were obtained from three measurements per site (Figure 2) [12].

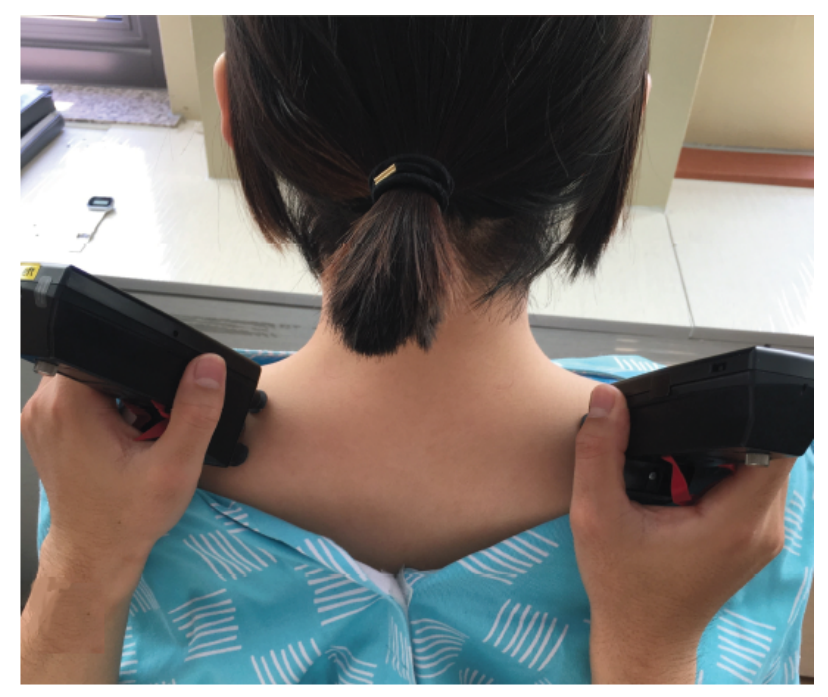

Figure 2. Static surface electromyography device and measurement sites (upper trapezius).

\section{Statistical analysis}

Statistical analysis was performed using PASW Statistics ver. 18.0 (IBM Co., Armonk, NY, USA). Test-retest reliability was calculated by ICCs. An independent sample t-test was conducted to compare the measurement variables between the two groups. Pearson correlation coefficients were used to determine the linear relationship between measured variables. All statistical significance levels were below $p=0.05$.

\section{Results}

\section{General characteristics of the subject}

As for general characteristics of the subjects, there were 22 subjects with cervical pain and 30 healthy adults who had participated in this study. The average values for the patients was $33.59 \pm 10.29$ years old for age, $161.60 \pm 4.49 \mathrm{~cm}$ for height, $55.77 \pm 9.38 \mathrm{~kg}$ for weight, and 5.65 for the VAS. The average values for the characteristics of healthy adults was $20.87 \pm 1.07$ years old for age, $160.72 \pm 4.67 \mathrm{~cm}$ for height, and $56.93 \pm 9.19 \mathrm{~kg}$ for weight. There was no significant difference in height and weight among the subjects, but there was a significant difference in age $(p<0.05)$ (Table 1$)$.

\section{Reliability analysis}

In the analysis of the test-retest reliability of the static sEMG and pressure gauge system in the subjects with neck

Table 1. General characteristics of subjects

$(\mathrm{N}=52)$

\begin{tabular}{lcccc}
\hline \multicolumn{1}{c}{ Group } & Age $(\mathrm{y})$ & Height $(\mathrm{cm})$ & Weight $(\mathrm{kg})$ & Visual Analog Scale \\
\hline Neck pain subjects $(\mathrm{n}=22)$ & $33.59(10.29)$ & $161.60(4.49)$ & $55.77(9.38)$ & 5.65 \\
Healthy adults $(\mathrm{n}=30)$ & $20.87(1.07)$ & $160.72(4.67)$ & $56.93(9.19)$ & - \\
$\mathrm{t}(p)$ & $-5.775(<0.001)$ & $-0.685(0.496)$ & $0.448(0.656)$ & - \\
\hline
\end{tabular}

Values are presented as mean (SD).

Table 2. Tests of Static sEMG and algometer values in subjects with neck pain and healthy adults-test-retest reliablility measurements

\begin{tabular}{llcc}
\hline \multirow{2}{*}{ Division } & \multirow{2}{*}{ Measuring site } & \multicolumn{2}{c}{ Intraclass correlation coefficient } \\
\cline { 3 - 4 } & & Neck pain subjects $(\mathrm{n}=22)$ & Healthy adults $(\mathrm{n}=30)$ \\
\hline \multirow{2}{*}{ Static sEMG } & Left & 0.867 & 0.861 \\
\multirow{2}{*}{ Algometer } & Right & 0.848 & 0.815 \\
& Left & 0.977 & 0.959 \\
& Right & 0.979 & 0.956 \\
\hline
\end{tabular}

sEMG: surface electromyography. 
Table 3. Differences in NDI, static sEMG, and pressure

$(\mathrm{N}=52)$

\begin{tabular}{|c|c|c|c|c|c|c|}
\hline \multirow{2}{*}{ Variable } & \multirow{2}{*}{$\begin{array}{l}\text { Neck Pain Subjects } \\
\qquad(\mathrm{n}=22)\end{array}$} & \multirow{2}{*}{$\begin{array}{l}\text { Healthy adults } \\
\qquad(\mathrm{n}=30)\end{array}$} & \multirow{2}{*}{ Mean } & \multicolumn{2}{|c|}{$95 \%$ confidence interval } & \multirow{2}{*}{$\mathrm{t}(p)$} \\
\hline & & & & Minimum & Maximum & \\
\hline NDI & $12.55(5.22)$ & $3.90(2.76)$ & -8.65 & -11.14 & -6.15 & $-7.081(<0.001)$ \\
\hline Static sEMG & $6.50(3.27)$ & $3.48(1.48)$ & -3.02 & -4.55 & -1.49 & $-4.039(<0.001)$ \\
\hline Algometer & $104.28(31.50)$ & $72.39(24.01)$ & -31.89 & -47.34 & -16.43 & $-4.145(<0.001)$ \\
\hline
\end{tabular}

Values are presented as mean (SD).

NDI: Neck Disability Index, sEMG: surface electromyography.

pain and healthy adults, the reliability of the static sEMG and pressure gauge showed ICC values of 0.815-0.979, which indicated a high level of reliability (Table 2 ).

Comparison of the Neck Disability Index, static surface electromyography, and pressure difference

There was a significant difference in NDI, static sEMG, and pressure gauge system values between persons with neck pain and healthy adults $(p<0.05)$ (Table 3$)$.

\section{Correlation analysis}

The VAS showed a statistically significant correlation with the static sEMG $(\mathrm{r}=0.540)$ and the pressure gauge system $(\mathrm{r}=0.543)$. The NDI was significantly correlated with static sEMG $(\mathrm{r}=0.380)$ and pressure gauge $(\mathrm{r}=0.615)$. Table 4 shows that there was no statistically significant correlation between the static sEMG and the pressure threshold.

\section{Discussion}

In order to evaluate the usefulness of static sEMG in persons with neck pain, this study examined a total of $52 \mathrm{sub}-$ jects, which included 22 subjects with neck pain and 30 healthy subjects. The reliability of the test method was verified by analyzing the difference of the static sEMG and pressure threshold test of all the subjects, and as the general characteristics of the patients with neck pain and healthy adults, VAS, NDI, static sEMG results were obtained, the purpose of this study was to investigate whether if static sEMG is a useful tool for measuring pain levels.

Results showed that the test-retest reliability of the static sEMG and pressure gauge system was $\mathrm{ICC}=0.815-0.867$, indicating high reliability, and it was confirmed that the pressure gauge system was a highly reliable evaluation tool with ICC $=0.956-0.979$. The results of the study on the reliability of the static sEMG were similar to the results of previous studies.
Table 4. Correlation of VAS, NDI, cervical active joint range, static sEMG, and pressure

\begin{tabular}{lcc}
\hline Variable & Static sEMG $(\mathrm{n}=52)$ & Pressure gauge meter $(\mathrm{n}=52)$ \\
\hline VAS & $0.540^{*}$ & $0.543^{*}$ \\
NDI & $0.380^{*}$ & $0.615^{*}$ \\
Algometer & 0.048 & - \\
\hline
\end{tabular}

VAS: Visual Analog Scale, NDI: Neck Disability Index, sEMG: surface electromyography.

${ }^{*} p<0.05$ : Pearson correlation.

Dedering et al. [19] stated that the use of EMG to examine for erector spinae muscle fatigue in subjects with low back pain had a high test-retest reliability of $\mathrm{ICC}=0.85$. A study by Cid et al. [20] found that the reliability of the EMG results for maximal contraction activity of the trapezius muscle in subjects with neck pain and healthy adults was high with ICC $=0.97$ and also suggested that sEMG is helpful in examining muscle contraction activities.

In addition, Mohseni Bandpei et al. [10] described static sEMG as a useful technique for distinguishing between back pain patients and healthy subjects in the assessment of muscle fatigue using sEMG for patients with back pain and healthy subjects.

The results of this study suggest that static sEMG may help to provide prompt and accurate information in the initial assessment of pain in the evaluation of pain according to muscular electrical resistance.

Fejer and Hartvigsen [21] stated that the percentage of patients with neck pain was $34.6 \%$ at $21-49$ years of age, $27.3 \%$ at $50-71$ years of age, and increased to $19.8 \%$ at $18-29$ years of age with a rate of $16.4 \%$ at $30-44$ years of age. This is similar to the age of the subjects in this study. Static sEMG signals were signicantly increased to twice the amount in subjects with neck pain compared to healthy adults $(p<0.05)$. Ambroz et al. [7] measured the muscle activity patients with chronic back pain using static sEMG and found that it was 3 times higher. In addition, a study by Nederhand et al. [22] 
was conducted to investigate the relationship between the upper traps muscle activity of patients with neck dysfunction and normal subjects using sEMG. The mean difference between the subjects was 0.8 for static activity and 3.9 to 8.1 for dynamic activity. The patients with neck disability showed higher scores on muscle activity. Similar results were obtained in this study.

Bittmen and Cram [23] suggested that since static sEMG measures the tone of muscles, it can objectively document muscle spasm or other muscle anomalies in the pain area, and it is suggested that muscle relaxation or stretching exercises can be proposed as a technique to treat muscle spasms.

Significant increases in pressure thresholds were observed in subjects with neck pain compared with healthy adults $(p<0.05)$. Cho et al. [24] showed that the pressure threshold of the patient over 12 months was significantly higher than that of the patients within 12 months, and the pain threshold was significantly higher in patients with pain compared to healthy subjects.

Ashton-Miller et al. [25] have shown that pain and decreased muscle strength leads to a decrease in proprioception, leading to a rise in pressure threshold, which is consistent with our study.

The purpose of this study was to analyze the relationship between static sEMG, pressure thresholds, VAS, and NDI in order to investigate the correlation between static sEMG and pain assessment tools.

One of the most common and useful methods of evaluating the neck pain is the VAS and the NDI, which were significantly correlated with the parameters of the static sEMG and the pressure thresholds. Castro Sánchez et al. [26] found that the pressure threshold value also changed corresponding to the VAS before and after the intervention in the study of the effects of acupuncture treatment on pressure tenderness threshold, quality of life, pain intensity, and depression in patients with myofascial pain syndrome. In addition, in Korea, it has been reported that there was a significant correlation between pressure thresholds of the painful muscle with the VAS ( $\mathrm{r}=-0.175)$ [25]. A study by Oliveira et al. [27] showed that there was a strong linear correlation between the VAS and static sEMG when some functions were analyzed in order to find an objective sEMG equipment that could be related to the lower extremity. The area of the EMG signal was positively correlated with the intensity of the electrical stimulation causing the pain $(\mathrm{r}=0.99)$, and the EMG signal was correlated with subjective pain. Although the measurement sites were different, the results were sim- ilar to the results of this study.

In the study by Jung and Kim [28], muscle contraction of the left and right trapezius muscles were significantly correlated with cervical dysfunction, as measured by a comparison of cervical dysfunction and EMG muscle contraction $(p<0.05)$. These results are consistent with the hypothesis that muscle weakness is caused by sustained epidemiological stress, weakening muscle strength increases the structural instability of the neck, and is associated with pain in the neck. There was a correlation between the state of the muscle signal measured by sEMG and the NDI, which may indicate pain [28]. However, this study did not show a significant correlation in the relationship between static sEMG and algometer results. Sommerich et al. [29] showed that even if sEMG electrodes were properly positioned on the specific muscle to be measured, if the electrode was placed so that it spanned the nerve distribution point of the muscle, it is considered that there may have been a problem associated with other locations. Therefore, even if the pain area was measured by static sEMG, it is considered that the pain threshold is not correlated with the pressure threshold, and the number of samples is small. Therefore, further studies on this are warranted on this subject.

This study had some limitations. The number of subjects in the study population was relatively low, and it is difficult to apply the research results the general population since the results had been obtained from patients with neck pain, and there were limitations in the ability to control various lifestyle factors (habits, psychological state, exercise habits) that could have affected the research result. In addition, it was not easy to fixate the static EMGs and therefore, it was not possible to measure the dynamic variables of the subjects. In the future, these limitations should be addressed, and pain evaluation and analysis study of static sEMG in dynamic tasks and various pre- and post-interventional studies are necessary.

The purpose of this study was to investigate the clinical usefulness of static sEMG in evaluating pain objectively and to provide the basic data of static sEMG equipment for persons with neck pain and healthy adults. It was confirmed that static sEMG is a reliable tool that can be useful in verifying the patient's pain level.

\section{Conflict of Interest}

The authors declared no potential conflicts of interest with respect to the authorship and/or publication of this 
article.

\section{References}

1. Haldeman S, Carroll L, Cassidy JD, Schubert J, Nygren Å. The bone and joint decade 2000-2010 task force on neck pain and its associated disorders. Eur Spine J 2008;17(Suppl 1):5-7.

2. Kim KY, Kim SY. The effect of lower trapezius strengthening exercises on pain, disability, cervical range of motion and strength of lower trapezius in patients with unilateral neck pain : a controlled randomized trial. Phys Ther Korea 2015;22:58-68.

3. DeVocht JW, Gudavalli K, Gudavalli MR, Xia T. Novel electromyographic protocols using axial rotation and cervical flexion-relaxation for the assessment of subjects with neck pain: a feasibility study. J Chiropr Med 2016;15:102-11.

4. Neblett R. Surface electromyographic (SEMG) biofeedback for chronic low back pain. Healthcare (Basel) 2016;4:E27.

5. Regence. Surface Electromyography (SEMG) including paraspinal SEMG [Internet]. Washington: Regence, 2018 [cited 2018 Jun 1]. Available from: http://blue.regence.com/medicare/med/ m-med73.pdf

6. Pullman SL, Goodin DS, Marquinez AI, Tabbal S, Rubin M. Clinical utility of surface EMG: report of the therapeutics and technology assessment subcommittee of the American Academy of Neurology. Neurology 2000;55:171-7.

7. Ambroz C, Scott A, Ambroz A, Talbott EO. Chronic low back pain assessment using surface electromyography. J Occup Environ Med 2000;42:660-9.

8. Girasol CE, Dibai-Filho AV, de Oliveira AK, de Jesus Guirro RR. Correlation between skin temperature over myofascial trigger points in the upper trapezius muscle and range of motion, electromyographic activity, and pain in chronic neck pain patients. $\mathrm{J}$ Manipulative Physiol Ther 2018;41:350-7.

9. Tsang SMH, Szeto GPY, Xie YF, Lee RYW. Association of electromyographic activation patterns with pain and functional disability in people with chronic neck pain. Eur J Appl Physiol 2018;118:1481-92.

10. Mohseni Bandpei MA, Rahmani N, Majdoleslam B, Abdollahi I, Ali SS, Ahmad A. Reliability of surface electromyography in the assessment of paraspinal muscle fatigue: an updated systematic review. J Manipulative Physiol Ther 2014;37:510-21.

11. Neblett R, Brede E, Mayer TG, Gatchel RJ. What is the best surface EMG measure of lumbar flexion-relaxation for distinguishing chronic low back pain patients from pain-free controls? Clin J Pain 2013;29:334-40.

12. So YJ, Woo YK. Effects of smartphone use on muscle fatigue and pain and, cervical range of motion among subjects with and without neck muscle pain. Phys Ther Korea 2014;21:28-37.

13. Farooq MN, Mohseni-Bandpei MA, Gilani SA, Ashfaq M, Mahmood Q. The effects of neck mobilization in patients with chronic neck pain: a randomized controlled trial. J Bodyw Mov Ther 2018;22:24-31.

14. Ferreira-Valente MA, Pais-Ribeiro JL, Jensen MP. Validity of four pain intensity rating scales. Pain 2011;152:2399-404.

15. Song KJ, Choi BW, Kim SJ, Yoon SJ. Cross-cultural adaptation and validation of the Korean version of the neck disability index. J Korean Orthop Assoc 2009;44:350-9.

16. Lesimple C, Fureix C, Biquand V, Hausberger M. Comparison of clinical examinations of back disorders and humans' evaluation of back pain in riding school horses. BMC Vet Res 2013;9:209.

17. Lee NY, Kim SY. Comparison of immediate effects of pain, range of motion and treatment satisfaction on difference of applying joint mobilization levels in patients with acute mechanical neck pain. Phys Ther Korea 2015;22:50-60.

18. Park KN, Oh JS. Influence of wearing a brassiere on pain and EMG activity of the upper trapezius in women with upper trapezius region pain. J Phys Ther Sci 2014;26:1551-2.

19. Dedering A, Gnospelius A, Elfving B. Reliability of measurements of endurance time, electromyographic fatigue and recovery, and associations to activity limitations, in patients with lumbar disc herniation. Physiother Res Int 2010;15:189-98.

20. Cid MM, Januario LB, Zanca GG, Mattiello SM, Oliveira AB. Normalization of the trapezius sEMG signal - a reliability study on women with and without neck-shoulder pain. Braz J Phys Ther 2018;22:110-9.

21. Fejer R, Hartvigsen J. Neck pain and disability due to neck pain: what is the relation? Eur Spine J 2008; 17:80-8.

22. Nederhand MJ, IJzerman MJ, Hermens HJ, Baten CT, Zilvold G. Cervical muscle dysfunction in the chronic whiplash associated disorder grade II (WAD-II). Spine (Phila Pa 1976) 2000;25: 1938-43.

23. Bittman B, Cram JR. Surface electromyography: an electrophysiological alternative in pain management. Paper presented at: 11th Annual Scientific Meeting; 1992 Oct.

24. Cho SG, Seo JC, Choi DY, Kim YS. Effects of acupuncture on upper back myofascial pain and pain pressure threshold. J Korean Acupunct Moxibustion Med Soc 2001;18:1-10.

25. Ashton-Miller JA, Wojtys EM, Huston LJ, Fry-Welch D. Can proprioception really be improved by exercises? Knee Surg Sports Traumatol Arthrosc 2001;9:128-36.

26. Castro Sánchez AM, García López H, Fernández Sánchez M, Pérez Mármol JM, Aguilar-Ferrándiz ME, Luque Suárez A, et al. Improvement in clinical outcomes after dry needling versus myofascial release on pain pressure thresholds, quality of life, fatigue, pain intensity, quality of sleep, anxiety, and depression in patients with fibromyalgia syndrome. Disabil Rehabil 2018. doi: 10.1080/09638288.2018.1461259. [Epub ahead of print]

27. Oliveira IM, Machado ARP, Pereira AA, Andrade AO. Estimated features from surface EMG of the lower limb correlate with the subjective sensation of pain. Psychol Neurosci 2014;7:355-61.

28. Jung JY, Kim SS. The relationship between neck pain and psychological state in female office workers. J Korea CHUNA Manual Med Spine Nerves 2009;4:1-12.

29. Sommerich CM, Joines SM, Hermans V, Moon SD. Use of surface electromyography to estimate neck muscle activity. J Electromyogr Kinesiol 2000;10:377-98. 